\title{
Measurement of the point spread function of a pixelated detector array
}

\author{
Christian Ritzer ${ }^{*}$, Patrick Hallen, David Schug, Volkmar Schulz \\ From PSMR 2015: 4th Conference on PET/MR and SPECT/MR \\ La Biodola, Isola d'Elba, Italy. 17-21 May 2015
}

Department of Physics of Molecular Imaging Systems, Institute for Experimental Molecular Imaging, RWTH Aachen University, Aachen, Germany
In order to further understand the PET/MRI scanner of our group, we measured the point spread function of a preclinical scintillation crystal array with a pitch of $1 \mathrm{~mm}$ and a total size of $30 \mathrm{~mm} \sim 30 \mathrm{~mm} \sim 12 \mathrm{~mm}$. It is coupled via a lightguide to a dSiPM from Philips Digital Photon Counting, used on the TEK-setup. Crystal identification is done with a centre of gravity algorithm and the whole data analysis is performed with the same processing software as for the PET insert, giving comparable results. The beam is created with a 22 NA-Point-Source and a lead collimator, with $0.5 \mathrm{~mm}$ bore diameter. The algorithm sorted $62 \%$ of the coincidences into the correct crystal.

Published: 18 May 2015

doi:10.1186/2197-7364-2-S1-A10

Cite this article as: Ritzer et al: Measurement of the point spread function of a pixelated detector array. EJNMMI Physics 2015 2(Suppl 1):A10.

\section{SpringerOpen $^{\circ}$}

(c) 2015 Ritzer et al; licensee Springer. This is an Open Access article distributed under the terms of the Creative Commons Attribution License (http://creativecommons.org/licenses/by/4.0), which permits unrestricted use, distribution, and reproduction in any medium, provided the original work is properly cited. 